\title{
Inspection of Materials by Laser Based 3D Scanning
}

\author{
Rajesh S, Kalyan Kumar Reddy N, Madhu P, Tarun K
}

\begin{abstract}
D Scanning system plays a vital role in the field of engineering as well as for engineering purposes. The $3 D$ Scanner is capable of capturing the real world image and converting it into digitalized form and sends the digitalized form of information to the computer for the further procedures. It aims at the reverse engineering process in a variety of industries like production industry to pertain the existing components for the inspection of quality components and for the simulation engineering to replica the nature from the authentic world. It may also leads to decrease in the manufacturing costs and also it creates much ease for the inspection of the materials. The application of $3 D$ scanning is improved by reducing the cost of $3 D$ scanning device. In this manuscript we dealt with the preparation or making of the $3 D$ scanner system in a compact size with reduced cost in order to be effectively available to everyone and also to amplify the quality of the $3 D$ scanning system.
\end{abstract}

Keywords : 3D Scanning, Reverse Engineering, Laser Light scanning.

\section{INTRODUCTION}

3D scanning is the method for examine the real-world entity or surroundings to accumulate data on its form and perhaps its exterior. The composed data is used to create digital models. A 3D scanner is categorized based on numerous technologies; everyone has its own confines, compensation and expenditure. A lot of confines in this type of items is digitized are still present. For illustration, optical technology may well come across numerous complexity with glossy, reflective or transparent objects. For instance, manufacturing computed tomography scanning and structured-light 3D scanners is used to construct digital 3D models, without destructive testing. Composed 3D data is helpful for a extensive function.

3D scanners contribute to a number of traits with cameras. Similar to the majority cameras, it has cone like pasture of vision. It collects the information concerning surfaces that are not obscured. As camera gather shade information concerning surfaces inside its field of view. This permit the three dimensional position of each point in the picture is identified.

3D Laser Scanning can generally be categorized into three main group:

Revised Manuscript Received on December 15, 2019.

* Correspondence Author

Rajesh $\mathbf{S}^{*}$, Department of Mechanical Engineering, Kalasalingam Academy of Research and Education, Krishnankoil, India. Email: s.rajesh@klu.ac.in

Kalyan Kumar Reddy N, Department of Mechanical Engineering, Kalasalingam Academy of Research and Education, Krishnankoil, India.

Madhu P, Department of Mechanical Engineering, Kalasalingam Academy of Research and Education, Krishnankoil, India.

Tarun K, Department of Mechanical Engineering, Kalasalingam Academy of Research and Education, Krishnankoil, India. $\checkmark \quad$ Laser triangulation

$\checkmark \quad$ Time of flight

$\checkmark$ Phase Shift

The laser techniques are used for independent comparisons and also used for developing the surface defects and other applications depending upon the kind of application we are employed with the component.

Laser triangulation is a technique implemented to measure or to capture the three dimensional image by triggering the light source where the camera is attached near to it. The light source like the laser and camera are both targeted to the object where it has to be scanned.

Time of flight laser scanners produce a pulse of laser light that is reflected off of the object to be scanned. It measures the value of the range where the system resolves the time of flight techniques to measure the distance between the camera and also the object where it is placed.

Phase shift laser scanners measure the distance of the light travelled through the phase difference of the light through the measured signals. When the phase difference between the light travelled is close to 2npie the cause of difficulty can occur. Hence the phase shift scanners are used to measure the distance of the light travelled to get the point cloud distances to be stored in the computer.

White Light Scanning is implemented to calculate the height of the object where the object is laid for the scanning. It is a device which is implemented to measure the surface heights using coherence light interferometry to measure the broadband white light illumination. Vertical scanning interferometry is an example for the low-coherence interferometry where it explodes the white light to measure the surface heights. If multiple sensors are used the software uses the known pattern and referencing between image angles to determine the 3D measurements. The disadvantage involved in the use of using the purely commercial scanners is that of the cost involved with it. It costs very higher that the expected cost.

\section{NEED OF USING THE LOW COST 3D SCANNING TOOLS}

3D scanning technology is used for many engineering applications. The 3D scanner involves the obtaining of the 3D model in the computer in the form of digital form. Reverse engineering concept plays a vital role in the $3 \mathrm{D}$ scanner for the quality inspection as well as it decrease the cost involved with the inspection. The following are the uses of the scanner technology.

- Reverse Engineering

- Inspecting the quality

- Computation of 3D Animation Character from Real world 


\section{Inspection of Materials by Laser Based 3D Scanning}

- Increase the speed of inspection

- Increasing accuracy working with complex parts and shapes

The reverse engineering implies creating 2D drawing by measuring the physical dimensions of the component. It is very difficult to inspect the complex components in the real world. For the inspection of those complex objects 3D scanner is used.

\section{WORKING PROCEDURE OF SCANNER}

The 3D scanner is used to scan the objects to get the dimensions of the object in the cloud point forms and gets this digitalized information to the computer. The kind of information will be in the form of point clouds and the collected information will get the dimensions of the scanned component. The hardware requirements of the scanner are as follows:-

Line Laser: Line laser can be of green or red color and it projects the laser in the form of a line instead of generating like points.

Camera: A good quality camera generally a web camera is used to get the image very clearly and depending on the quality of the camera the results will be accurate.

Motorized setup: For the inspection setup of the scanner we are going to use the step-up motor where the step-up motor divides the whole rotation of the inspection setup in several number of steps to get the accurate results of the scanned part. It results in getting accurate dimensions.

The working principle of the scanner is as follows:

The camera working will be the first step. It involves in identifying the $3 \mathrm{D}$ points of the object corresponds to the $2 \mathrm{D}$ points of the object that is being captured. Here the method uses the checkerboard pattern to calibrate the camera in the necessary points of the object to different orientations to get the corresponding 3D points of the object.

The calibration of the camera and the laser is the second step where the laser orientation should be less than 20 degrees.

The projection of line laser will be the final step where the laser helps to store the information to get the 3D point cloud data from the necessary laser projection.

Zhang (2000) [1] is the one who proposed the checkerboard pattern of calibrating the camera to get the point cloud data from where the laser orientation emits in two directional in order to be perfectly projected laser the checkerboard pattern is used.

Chang and Chen (2005) [2] in this study they have explained how to get the image of the welded objects in order to inspect the quality of the weld. It enhances the flexibility of the scanner in order to get the welded images and also in identifying the corresponding defects.

Yao (2005) [3] here the discussion takes place regarding the response time of the quality control of the products. The scanner along with the software like CAD gives the application of reverse engineering concept in order to get the accurate results of the scan.

Furukawa and Kawasaki (2005) [4] here they replaces the camera with a video projector. They gives an example of the structured light technology where the video projector and the lasers forms a stereo system the projector will be used as a substitute for the camera. Here the projector enhances the intrinsic parameters of the object to be scanned. In this paper two scanning methods are used. The first method involves keeping either the camera or the projector fixed and the other device moving. Since this method utilizes the information of multiple images, the precision of the camera positions improves, producing a better quality $3 \mathrm{D}$ reconstruction. The second method involves fixing each of them alternatively. In this method of scanned first the camera is fixed and the projector is moved and scan results are taken and then projector is fixed and camera is moved and the steps are repeated. This method has the advantage of covering wide area of scan.

Zhang and Huang (2006) [5] here they explain the use of high resolution of the cameras to get the scanned object in an accurate manner where he explains the principle with respect to the phase shift technique and also fringe pattern technique. They proposed a kind of camera which commences the image capturing at a speed of 120 frames per second where the image acquisition is around 40 frames per second to get the accurate image of the object to give the good results.

Lanman et al (2000) [6] here they proposed a concept of introducing the plane mirrors to obtain the qualitative 3D scanning image using the video projector. Here the system requires the plane mirrors to be in the orthogonal projection where the video projector has been laid over it to capture the image to get the accurate measurements of the scanned image.

Peiravi \& Taabbodi (2010) [7] states the use of the two lasers like green and red color. Both the colors are differentiated based on their difference in their wavelengths. Here we have studied about the Red and Green base line their focal lengths where using these parameters the quality of the scan can be greatly enhanced.

Martinez et al. (2010) [8] here they used the scanning technique in order to measure the heavy objects like meteorological applications to get the geometrical accuracy of the scanned objects and also for the tolerance control. The common alignment method is used to compare the accuracy of both the scanning systems. It uses computer aided design software CATIA V5 for converting point cloud data into Cad model and GEOMAGIC Studio for reverse engineering of scanned data into 3D surface reconstruction, Geometric Qualify software is used for inspection.

Martynov et al. (2011) [9] here they explained about the calibration of the video projection. Here the camera is calibrated like the method of sensor which easily senses the object to be objectified which uses only a single camera where it enables to see the projection of dots to measure the distance between the calibrated objected as well as the camera.

Park et al. (2011) [10] uses a hand-held 3D scanning technique to reconstruct $3 \mathrm{D}$ models of real objects. A sequence of images is captured from a hand-held.

\section{CONCLUSION}

From the study we have concluded that the $3 \mathrm{D}$ scanner is used to various engineering applications to create the shape of the object from the real world and converts it into the digital form and stores the gathered information to the computer. It is very widely used in gathering the information like shape, size and other dimensions of the objects and it can be used as an application of reverse engineering 
techniques.

It sometimes can also be used as a device used to detect the surface defects of the object which is to be scanned by the scanner but depending on the application to which it is employed the time depends on it. Many researchers have been done in order to enhance the use of scanner in various applications which greatly influences the Light scanning of the laser and the structured light scanning to get the accurate results which can be found as an application of reverse engineering process and other inspection purposes.

\section{REFERENCES}

1. ZHANG Z, "A flexible new technique for camera calibration." IEEE Trans. Pattern Anal. Mach. Intell., pp 1330-1334(2000).

2. C.L. Chang, Y.H. Chen, "Measurement of Fillet weld by $3 \mathrm{~d}$ laser scanning system", International Journal of Advance Manufacturing Technology, Vol. 25, pp.466 - 470,(2005).

3. A.W.L. Yao, "Applications of 3D scanning and reverse engineering techniques for quality control of quick response products", International Journal of Advance Manufacturing Technology , Vol. 26, pp. 1284-1288,(2005).

4. Ryo Furukawa and Hiroshi Kawasaki, "Uncalibrated multiple image stereo system with arbitrarily movable camera and Projector for wide range scanning."IEEE 3DIM '05 Proceedings of the Fifth International Conference on 3-D Digital Imaging and Modelling, pp. 302-309 (2005).

5. Song Zhang and Peisen S. Huang, "High-resolution, real-time three-dimensional shape measurement." Optical engineering, 123601, Vol. 25 (2006).

6. Lanman Et al. , "Surround Structured Lighting for Full Object Scanning." 3DIM '07 Proceedings of the Sixth International Conference on 3-D Digital Imaging and Modeling, pp. 107-116 (2007).

7. Ali Peiravi, Behrai Taabbodi, "A Reliable 3D Laser Triangulation-based Scanner with a New Simple but Accurate Procedure for Finding Scanner Parameters" Journal of American Science, Vol. 6, pp. 80-85, (2010).

8. Martinez Et al. , "Analysis of laser scanning and strategies for dimensional and geometrical control" International Journal of Advance Manufacturing Technology, Vol. 46, pp. 621-629, (2010).

9. I. Martynov, J.-K. Kamarainen, and L. Lensu, "Projector calibration by inverse camera calibration," in Proceedings of the 17th Scandinavian conference on Image analysis, Berlin, Heidelberg, pp. 536-544, (2011).

10. S.Y. Park, J. Baek, J. Moon, "Hand-held 3D scanning based on coarse and fine registration of multiple range images", Machine Vision and Applications, Vol. 22, pp. 563-579, (2011).

11. Xu, Cancan, Guohao Dai, and Yi Hong. "Recent advances in high-strength and elastic hydrogels for 3D printing in biomedical applications." Acta biomaterialia 95 (2019): 50-59.

12. Zuo, Zibo, Jian Gong, Yulin Huang, Yijian Zhan, Ming Gong, and Lulu Zhang. "Experimental research on transition from scale 3D printing to full-size printing in construction." Construction and Building Materials 208 (2019): 350-360.

13. Kang, Kyung Seok, Chanhyuk Jee, Ji-Hong Bae, Hyo Jin Jung, and PilHo Huh. "Heat Capacity Variables of Thermoplastic Polyurethane for High-quality 3D Printing Resolution and Their Characteristics." Materials Letters (2019): 126698.

\section{AUTHORS PROFILE}

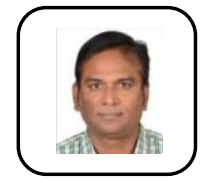

Dr. S. Rajesh, completed his B. E in Mechanical Engineering and M.E. (CAD) from Alagappa Chettiar Government College of Engineering and Technology, Karaikudi, Ph.D (Machining of Metal of Matrix Composites) from Kalasalingam Academy of Research and Education, Krishnankoil in the year 2014, and thesis highly recommended by both the examiners. In the year 2018, I have received DST - AMT project for the worth of Rs. 25,72,400 and completed one IEDC project for the worth of Rs. 1,00,000. He has published more than 25 Journal papers (Impact Factor and Scopus Indexed) and more than 40 International Conference papers. 